\title{
Modeling the shelter site location problem using chance constraints: A case study for Istanbul
}

\author{
Ömer Burak Kınay ${ }^{\mathrm{a}, 1}$, Bahar Yetis Kara ${ }^{\mathrm{b}, *}$, Francisco Saldanha-da-Gama ${ }^{\mathrm{c}}$, Isabel Correia ${ }^{\mathrm{d}}$ \\ a Department of Management Sciences, University of Waterloo, Waterloo, ON, Canada \\ ${ }^{\mathrm{b}}$ Department of Industrial Engineering, Bilkent University, Ankara, Turkey \\ ${ }^{\mathrm{c}}$ Departamento de Estatística e Investigação Operacional / Centro de Matemática, Aplicaes Fundamentais e Investigao Operacional, Faculdade de Ciências, \\ Universidade de Lisboa, Campo Grande 1749-016, Lisboa, Portugal \\ ${ }^{\mathrm{d}}$ Departamento de Matemática / Centro de Matemática e Aplicações, Faculdade de Ciências e Tecnologia, Universidade Nova Lisboa, Caparica 2829-516, \\ Portugal
}

\section{A R T I C L E I N F O}

\section{Article history:}

Received 7 December 2017

Accepted 5 March 2018

Available online 11 March 2018

\section{Keywords:}

Location

Humanitarian logistics

Shelter site location

Probabilistic programming

Approximations

\begin{abstract}
A B S T R A C T
In this work, we develop and test a new modeling framework for the shelter site location problem under demand uncertainty. In particular, we propose a maxmin probabilistic programming model that includes two types of probabilistic constraints: one concerning the utilization rate of the selected shelters and the other concerning the capacity of those shelters. By invoking the central limit theorem we are able to obtain an optimization model with a single set of non-linear constraints which, nonetheless, can be approximated using a family of piecewise linear functions. The latter, in turn, can be modeled mathematically using integer variables. Eventually, an approximate model is obtained, which is a mixed-integer linear programming model that can be tackled by an off-the-shelf solver. Using the proposed reformulation we are able to solve instances of the problem using data associated with the Kartal district in Istanbul, Turkey. We also consider a large-scale instance of the problem by making use of data for the whole Anatolian side of Istanbul. The results obtained are presented and discussed in the paper. They provide clear evidence that capturing uncertainty in the shelter site location problem by means of probabilistic constraints may lead to solutions that are much different from those obtained when a deterministic counterpart is considered. Furthermore, it is possible to observe that the probabilities embedded in the probabilistic constraints have a clear influence in the results, thus supporting the statement that a probabilistic programming modeling framework, if appropriately tuned by a decision maker, can make a full difference when it comes to find good solutions for the problem.
\end{abstract}

(c) 2018 Elsevier B.V. All rights reserved.

\section{Introduction}

Turkey is a country much vulnerable to natural disasters. Since the beginning of the 20th century more than half-million homes have been destroyed (Ozmen, Nurlu, Kuterdem, \& Temiz 2010) due to different kinds of disasters (e.g, earthquakes, landslide, flood). Among these sudden events, earthquakes are the ones with the most severe consequences being responsible for approximately $80 \%$ of the destroyed homes.

In recent years, much literature has emerged in the topic of disaster management operations. The reader can refer to Altay and Green (2006), Caunhye, Nie, and Pokharel (2012), Galindo

\footnotetext{
* Corresponding author.

E-mail address: bkara@bilkent.edu.tr (B. Yetis Kara).

1 This research was initiated when the author was at Department of Industrial Engineering, Bilkent University, Ankara, Turkey.
}

and Batta (2013), and Hoyos, Morales, and Akhavan-Tabatabaei (2015) and to the references therein. The review paper by Galindo and Batta (2013) emphasizes the role of Operations Research and stochastic modeling in that topic.

The existing work in disaster operations management can be classified according to four main categories namely, the following ones: (i) mitigation; (ii) preparedness; (iii) response; and (iv) recovery. The pre-disaster operations are related with categories (i) and (ii) while categories (iii) and (iv) refer to pos-disaster operations. In particular, mitigation refers to the actions taken in order to prevent and mitigate the consequences of a disaster. The preparedness phase involves the elaboration of plans to provide a more efficient response when a disaster occurs. The response phase starts immediately after the event and aims to quickly provide the affected people with relief goods such as water, food, medical care, and shelter. Finally the recovery phase takes usually much time and aims to recover all the damaged (infra)structures 
in order to ensure the normal functioning of the affected region.

When a disaster results in people losing their homes it is necessary to accommodate such people in temporary shelter areas until the disaster recovery process is finished. Therefore the problem that consists of selecting new temporary shelter areas is one of the fundamental facility location problems in the preparedness phase for disaster relief. The problem is known in the literature as the shelter site location problem (Kılc1, Kara, \& Bozkaya, 2015).

Most of the developed countries have specific organizations that are responsible for locating shelter areas and providing the emergency supplies to the affected people. For instance, in the earthquake occurred in central Italy in August 24, 2016, the Italian Civil Department (Dipartimento Protezione Civile, http: //www.protezionecivile.gov.it) took the lead in terms of sheltering.

In the particular case of Turkey that we consider in more detail in this work, it is up to the Red Crescent (http://www.ifrc.org/), namely to its Turkish branch (Tirk Kizlayl, http://www.kizilay.org.tr/) to organize this type of response. In regions where the probability of a catastrophe is high (e.g. Istanbul) the organization selects possible shelter locations before a disaster occurs. This is accomplished by considering several criteria for ranking potential shelter locations (e.g. distance to healthcare institutions, electrical infrastructure, sanitary system, etc). The reader should refer to Kilc1 et al. (2015) for all the details. When a candidate shelter location is identified, it is classified between 0 and 1 with respect to each of these criteria. The final weight of a candidate location results from computing a convex combination of all its scores. Finally, all potential shelters locations can be sorted non-increasingly according to their weights. When a disaster occurs, the Turkish Red Crescent uses the list of locations induced by the above sorting for sequentially deciding about the shelters to construct. The selection process proceeds until the sheltering made available is enough for accommodating all the affected people.

The above methodology can be easily improved by capturing other aspects. This fact was observed by Kilc1 et al. (2015) who considered the following additional aspects: (i) distance between the potentially affected people and the shelter areas; (ii) utilization rate of the shelters; and (iii) pairwise utilization difference of the open shelter areas. In order to accommodate these issues, Kilc1 et al. (2015) proposed an integer linear programming formulation for the shelter site location problem. This is a model aiming at maximizing the minimum weight of the shelters to open while deciding about the assignment of the population areas (or regions, zones, et cetera) to those shelters and simultaneously ensuring a minimum threshold for their utilization rate. The authors also consider a shelter pairwise balancing constraint.

Unfortunately, neither can the occurrence of a disaster nor its consequences be predicted in most of the cases (e.g. earthquakes). Moreover, its destruction level may vary significantly according to intensity, location and duration; hardly can the impact be known in advance. Consequently, the amount of sheltering needed may vary significantly.

Since the selection of the candidate locations for the shelters is made a priori, it is important to take uncertainty into account when doing it. One possibility for dealing with this uncertainty is to plan for covering all the demand whatever it may turn out to be. However, this can easily lead to a so-called "fat" solution and thus a very costly one in which the installed capacity may be significantly above the actual needs. Alternatively, one may consider uncertain demand and keep imposing "hard" capacity constraints ensuring that the installed capacity should hold for all possible scenarios. In this case, we would have to plan for the worst-case scenario, which may be a very unlikely one (as it surely is in the case of disaster events). Another possibility that emerges and that motivates this work is to consider a kind of "service level" constraints ensuring that with some (high) probability, each open shelter can cope with the demand of those districts previously assigned to it. In other words, we can consider a chance-constrained model for the problem. This allows capturing a finite set of scenarios with each one calling for some (predictable) amount of sheltering. Furthermore, if necessary, by using historical data, it is reasonable to assume that the probability associated to each scenario can be accurately estimated. In this case, the shelter areas are selected in such a way that with prespecified probabilities, (i) the total demand does not exceed the shelters' capacity; and (ii) the utilization rate of the shelters is not below a given threshold.

As we can conclude by the results obtained and that will be reported later in the paper, this type of model may provide good trade-off solutions for the shelter site location problem under demand uncertainty. Moreover, under some mild assumptions, the model can be approximated by a deterministic mixed-integer nonlinear programming problem for which an approximation approach can be devised. In particular, we can consider a mixed-integer linear programming optimization model that can be tackled by a general-purpose solver.

The remainder of this paper is organized as follows: in Section 2 we review the most relevant literature related with our work. In Section 3, we present an optimization model for the problem. Section 4 focuses on the development of an approximate mixed-integer linear programming model. The results of the computational tests performed using this model are reported in Section 5. The paper ends with an overview of the work done and also with some guidelines for future research.

\section{Literature review}

The application of OR/MS models and methods to disaster operations management is not new (Altay \& Green 2006; Galindo \& Batta 2013). Within this field, humanitarian logistics has emerged as an important topic in which much research has been done, which is attested by Kovács and Spens (2007), Ortuño et al. (2013), and Leiras, de Brito, Peres, Bertazzo, and Yoshizaki (2014), among others.

A relevant class of problems in the context of humanitarian logistics concerns location problems for disaster relief (e.g., Rawls \& Turnquist 2010). Kilc1 et al. (2015) classified such problems according to (i) an emergency medical location problem; (ii) a relief material warehouse location problem; (iii) a shelter site location problem. Most of the existing literature covers categories (i) and (ii). In this work, we focus on category (iii). In this case, the literature is scarce indicating that this is a family of problems whose study is much unexplored. Nevertheless, we can find some related literature as we detail next.

Sherali, Carter, and Hobeika (1991) studied a problem that consists of selecting a set of shelters to open together with an evacuation plan for automobiles that altogether minimize the evacuation time. The authors developed a non-linear mixedinteger programming formulation and developed a heuristic and an exact approach based upon a generalized Benders decomposition method. They presented tests using the network of a city in southeastern Virginia.

Alçada-Almeida, Tralhão, Santos, and Coutinho-Rodrigues (2009) considered a potential disaster triggered by fire in an urban area and proposed a multiobjective model for locating $p$ shelters together with the identification of evacuation routes. Data from the city of Coimbra, Portugal, was considered in that study. The work would be later extended by Coutinho-Rodrigues, Tralhão, and Alçada-Almeida (2012), where a multi-objective location-routing model was proposed for shelter site location and evacuation planning. An exogenous risk measure is considered for the evacuation paths and for the shelters. Such risk is included in some of the six 
objectives considered. The model aims at identifying the number and location of shelters as well as a set of primary and secondary evacuation routes.

Chanta and Sangsawang (2012) investigated a bi-objective model to determine the locations for at most $p$ shelters to serve a region suffering from a flood disaster. One objective concerns the minimization of the total weighted distance from each affected area to the closest shelter; the other one aims at maximizing the population that has a shelter within a prespecified distance (coverage objective). The proposed model is assessed using data from Bangkruai district in central Thailand.

Bayram, Tansel, and Yaman (2015) analyzed a shelter site location problem combined with evacuation traffic management. The goal is to find a solution that minimizes the total evacuation time in case of a disaster. The proposed models were tested using networks available in the literature as well as the Istanbul road network.

Chen and Yu (2016) considered a median-type problem setting a maximum number of facilities to open in the context of emergency medical service (EMS). The goal is to ensure an effective EMS after a disaster. A Lagrangean relaxation based approach is proposed for tackling the mathematical model proposed. The methodology is tested using a set of randomly generated instances.

The above mentioned works assume a deterministic setting in which the data is fully known in advance and is not subject to any type of uncertainty (e.g., data is estimated using historical series). However, when planning for mitigating the consequences of a disaster it is often the case that the use of deterministic information oversimplifies the problem. This is the case, for instance, when the consequences of a disaster may vary significantly. A quantitative approach for better hedging against such uncertainty requires it to be explicitly considered in a model. In this case, historical data can be used to define a set of possible scenarios (for instance in terms of the population that need sheltering) as well as for estimating their occurrence probabilities.

Although stochastic facility location problems have been studied for many years (the reader can refer to Correia and Saldanha-da-Gama (2015) and to the references therein) to the best of the authors' knowledge, the first paper investigating a stochastic shelter site location problem is due to $\mathrm{Li}$, Jin, and Zhang (2011), who focused on disasters caused by hurricanes. The authors proposed a two-stage stochastic programming formulation for the problem that consists of locating a set of shelters (firststage decision) and distributing the resources and the affected populations among the shelters (second-stage decision). Data from the Gulf Coast region of the USA was considered to test the developed solution algorithm which is a decomposition approach based upon the L-shaped method.

Li, Nozick, Xu, and Davidson (2012) proposed a bilevel optimization model for selecting a set of shelter locations that is robust for a range of hurricanes scenarios. In particular, the authors consider possible disruptions at the shelters. The upper-level problem is a two-stage stochastic programming problem defining the location-allocation problem related with the shelters; The lower-level problem focus on the behavior of the evacuees when it comes to choose an evacuation route. With this purpose, a socalled dynamic user equilibrium model is considered. The overall goal is to minimize the total system cost. Heuristic algorithms are developed for finding feasible solutions to the problem. A case study in North Carolina, USA, is presented.

Bayram and Yaman (2018a) investigated a two-stage stochastic approach whose objective is the minimization of the total evacuation time. In the first stage, at most $p$ shelters are to be located. The allocation of affected populations to the shelters and to the routes that were not disrupted is made in the second phase. By reformulating the problem as a second order conic mixed-integer programming model the authors can make use of general purpose solver for tackling it. In a following work, Bayram and Yaman (2018b) consider the same setting; however, now they introduce a scenario-based two-stage stochastic evacuation planning model and proposed an exact algorithm based on Benders decomposition to solve the second stage which is a second-order cone programming problem.

As mentioned before, in the current paper we consider a different perspective when it comes to capturing uncertainty in the shelter site location problem: we allow having a service level below $100 \%$ for one or several scenarios and we consider "soft" constraints for the minimum utilization rate of the selected shelters. This is accomplished by considering probabilistic constraints both for the installed capacity and for the utilization rates.

The integration of probabilistic programming (Charnes \& Cooper, 1959; Prékopa, 1995) with Location Analysis has its roots in the seminal paper by ReVelle and Hogan (1989) focusing the location of emergency facilities see (Birge \& Louveaux, 2011; Correia \& Saldanha-da-Gama, 2015; Snyder, 2006). In fact, like in that work, most of the related literature emerges from the need to locate emergency facilities. This is the case with the paper by Beraldi, Bruni, and Conforti (2004) aiming at designing a robust emergency medical service. The authors developed a chanceconstrained model for determining where to locate facilities as well as the number of emergency vehicles to assign to each facility. The goal is to ensure a certain reliable level of service at minimum cost. More recently, Zhang and Li (2015) proposed a model with chance constraints for designing an emergency medical service assuming uncertain demand. The probabilistic constraints are then approximated by second order conic inequalities rendering a model tractable by an off-the-shelf solver.

Bilsel and Ravindran (2011) proposed a multi-objective chanceconstrained model for hedging against uncertainty in a supplier selection problem. In this case, the set of facilities (suppliers) is not a specific decision to make since they exist and are known in advance. However, not all are necessarily used in a solution. Accordingly, the allocation decisions to make induce the facilities to select. Uncertainty is associated with demand, capacity at suppliers and variable costs. Assuming that the stochastic parameters follow a normal distribution, the authors are able to linearize the non-linear deterministic equivalent. That assumption is then relaxed and a more general setting is considered.

In the context of a bio-terrorist attack, Murali and Ordónez (2012) studied a chance-constraint model for locating emergency facilities. By assuming a lognormal distribution for the demand the authors were able to linearize the chance constraints. A heuristic approach was developed in that work for the approximate problem.

Hong, Lejeune, and Noyan (2015) proposed a model for a stochastic pre-disaster relief network design problem. The model determines the sizes and locations of the response facilities as well as the amount of emergency supplies to be stocked in order to assure some network reliability. The model includes a chanceconstraint that establishes a high probability in the demand satisfaction.

Elçi, Noyan, and Bülbül (2016) studied a post-disaster twoechelon network design problem. In the first echelon a local distribution center receives the relief supplies and sends them to the points of distribution while in the second echelon the demand points receive the relief supplies from the points of distribution. The authors propose a model that considers equity and accessibility measures and takes into account the uncertainty associated with the demands and with the transportation network structure after a disaster. The demand satisfaction constraints are modeled as chance constraints. 
Table 1

Synthesis of the related literature.

\begin{tabular}{|c|c|c|c|c|c|c|c|c|}
\hline Study & Demand & $\begin{array}{l}\text { Location } \\
\text { decisions }\end{array}$ & $\begin{array}{c}\text { Allocation } \\
\text { pattern }\end{array}$ & $\begin{array}{l}\text { Closest } \\
\text { assignment } \\
\text { constraints }\end{array}$ & $\begin{array}{l}\text { Chance } \\
\text { con- } \\
\text { straints }\end{array}$ & $\begin{array}{l}\text { Service } \\
\text { level }\end{array}$ & Objective & Underlying setting \\
\hline $\begin{array}{l}\text { Sherali et al. } \\
\text { (1991) }\end{array}$ & Deterministic & $\boldsymbol{v}$ & Single & $x$ & $x$ & $x$ & Evacuation time (min) & Evacuation planning \\
\hline $\begin{array}{l}\text { Beraldi et al. } \\
\text { (2004) }\end{array}$ & Stochastic & $\boldsymbol{v}$ & Multiple & $x$ & $\boldsymbol{v}$ & $\checkmark$ & Total cost $(\mathrm{min})$ & $\begin{array}{l}\text { Emergency medical } \\
\text { services }\end{array}$ \\
\hline $\begin{array}{l}\text { Alçada-Almeida } \\
\text { et al. (2009) }\end{array}$ & Deterministic & $\boldsymbol{v}$ & Single & $x$ & $x$ & $\checkmark$ & $\begin{array}{l}\text { Distance \& risk \& time } \\
\quad(\text { min })\end{array}$ & $\begin{array}{l}\text { Shelter site location \& } \\
\text { evacuation paths }\end{array}$ \\
\hline $\begin{array}{l}\text { Rawls and } \\
\text { Turnquist } \\
\text { (2010) }\end{array}$ & Stochastic & $\boldsymbol{v}$ & Single & $x$ & $x$ & $\checkmark$ & Total cost (min) & $\begin{array}{l}\text { Prepositioning of } \\
\text { emergency supplies }\end{array}$ \\
\hline $\begin{array}{l}\text { Bilsel and } \\
\text { Ravindran } \\
\text { (2011) }\end{array}$ & Stochastic & $x$ & Single & $x$ & $\boldsymbol{v}$ & $\checkmark$ & Total cost (min) & Supplier Selection \\
\hline Li et al. (2011) & Stochastic & $\checkmark$ & Single & $x$ & $x$ & $\checkmark$ & Total cost (min) & Shelter site loocation \\
\hline $\begin{array}{l}\text { Chanta and } \\
\text { Sangsawang } \\
\text { (2012) }\end{array}$ & Deterministic & $\boldsymbol{v}$ & Single & Via Objective & $x$ & $\checkmark$ & $\begin{array}{l}\text { Coverage (max); total } \\
\text { distance (min) }\end{array}$ & $\begin{array}{l}\text { Shelter site location \& } \\
\text { evacuation paths }\end{array}$ \\
\hline $\begin{array}{l}\text { Coutinho- } \\
\text { Rodrigues et al. } \\
\text { (2012) }\end{array}$ & Deterministic & $\boldsymbol{v}$ & Single & $x$ & $x$ & $\checkmark$ & $\begin{array}{l}\text { Distance \& risk \& time } \\
\quad(\min )\end{array}$ & $\begin{array}{l}\text { Shelter site location \& } \\
\text { evacuation paths }\end{array}$ \\
\hline Li et al. (2012) & Stochastic & $\boldsymbol{v}$ & Single & $x$ & $x$ & $\checkmark$ & $\begin{array}{l}\text { Traveled distance \& } \\
\text { unmet demand ( } \mathrm{min})\end{array}$ & Shelter site location \\
\hline $\begin{array}{l}\text { Murali and } \\
\text { Ordónez (2012) }\end{array}$ & Stochastic & $\boldsymbol{v}$ & Single & $x$ & $\boldsymbol{v}$ & $\checkmark$ & Coverage (max) & $\begin{array}{l}\text { Location of distribution } \\
\text { centers for bio-terror } \\
\text { attack }\end{array}$ \\
\hline $\begin{array}{l}\text { Bayram and } \\
\text { Yaman } \\
(2018 a, b)\end{array}$ & Stochastic & $\checkmark$ & Single & $\checkmark$ & $x$ & $\checkmark$ & Evacuation time (min) & $\begin{array}{l}\text { Shelter site location \& } \\
\text { evacuation routes }\end{array}$ \\
\hline $\begin{array}{l}\text { Bayram et al. } \\
\text { (2015) }\end{array}$ & Deterministic & $\boldsymbol{v}$ & Single & $\boldsymbol{v}$ & $x$ & $\checkmark$ & Travel time (min) & $\begin{array}{c}\text { Shelter site location \& } \\
\text { evacuation routes }\end{array}$ \\
\hline Hong et al. (2015) & Stochastic & $\boldsymbol{v}$ & Single & $x$ & $\boldsymbol{v}$ & $\checkmark$ & Total cost (min) & $\begin{array}{r}\text { Pre-disaster relief } \\
\text { network design }\end{array}$ \\
\hline Kılcı et al. (2015) & Deterministic & $\checkmark$ & Single & $\boldsymbol{v}$ & $x$ & $\checkmark$ & $\begin{array}{l}\text { Minimun weight of } \\
\text { facilities (max) }\end{array}$ & Shelter site location \\
\hline $\begin{array}{l}\text { Zhang and Li } \\
\text { (2015) }\end{array}$ & Stochastic & $\checkmark$ & Single & $x$ & $\boldsymbol{v}$ & $x$ & Total cost (min) & $\begin{array}{l}\text { Location of emergency } \\
\text { medical services }\end{array}$ \\
\hline $\begin{array}{l}\text { Chen and Yu } \\
\text { (2016) }\end{array}$ & Deterministic & $\boldsymbol{v}$ & Single & $x$ & $x$ & $x$ & Total cost (min) & $\begin{array}{l}\text { Location of EMS } \\
\text { facilities }\end{array}$ \\
\hline Elçi et al. (2016) & Stochastic & $\boldsymbol{v}$ & Single & $x$ & $\boldsymbol{v}$ & $\checkmark$ & Accessibility (max) & $\begin{array}{l}\text { Location of distribution } \\
\text { centers }\end{array}$ \\
\hline This study & Stochastic & $\boldsymbol{r}$ & Single & $\boldsymbol{v}$ & $\boldsymbol{v}$ & $\checkmark$ & $\begin{array}{l}\text { Minimun weight of } \\
\text { facilities (max) }\end{array}$ & Shelter site location \\
\hline
\end{tabular}

Finally, we quote the paper by Lin (2009) to which some of our methodological developments are related. That author used probabilistic constraints to model service level in a single-source capacitated facility location model with uncertain demand. Two probability distributions were considered for the demand: Poisson and Normal. When the demand occurs according to a Poisson distribution the stochastic problem is equivalent to a deterministic single-source capacitated facility location problem. In turn, for Normal distributed demand, the stochastic problem is equivalent to a mixed-integer non-linear programming problem. Lower and upper bounds were then developed for its optimal value.

Table 1 pivots on the characteristics of this study and puts together all the main aspects of the aforementioned related literature explicitly. What emerges clearly from observing this table is that the shelter site location problem was never considered from a chance-constrained modeling framework perspective although it seems to make much sense as above explained. To the best of the authors' knowledge, our work is the first study of the shelter site location problem literature that considers chance constraints for capturing the stochastic nature of the demand under a disaster event. Besides it is also worth-noticing that we are considering service-level requirements combined with capacitated facilities and single-sourcing. In our case, evacuation decisions are implicit by using the closest assignment constraints. Finally, we are considering a maxmin type of objective function. Therefore, unlike other works, we do not aim at minimizing the total evacuation time.

\section{Shelter site location under uncertainty}

In this section, we introduce a chance-constrained model for the shelter site location problem. We start by presenting a deterministic version of the problem which will be extended and worked out in Section 3.2 by including the probabilistic constraints.

\subsection{Deterministic problem}

Our starting point is a deterministic model resulting from the one proposed by Kilcl et al. (2015).

As mentioned in the introductory section, in the particular case of Turkey (the case we are focusing on in this work) the Turkish Red Crescent ranks each potential sheltering location according to their weights which, in turn, are computed using several criteria (e.g. distance to healthcare institutions, electrical infrastructure, sanitary system, etc).

In terms of capacity, it is assumed that at least 3.5 square meters are allocated to each person in a shelter area. Additionally a shelter must have space assigned to facilities 
related with accessibility (roads), health, education, sanitation, and nutrition.

In their model, Kilcr et al. (2015) assumed that people living in the same district are all assigned to the closest open shelter. This is a means to help keeping the social structure of the society after a disaster.

Some assumptions of the model proposed by K1lc1 et al. (2015) that we also consider in this work are the following:

- The set of candidate locations for the shelters is known in advance. This set only includes candidate locations such that their distance to the nearest health center and to the nearest road is below maximum values previously decided for the accessibility to those infrastructures; in other words, instead of considering constrains (5) and (6) of K1lcl et al. (2015) we make a preprocessing and we only consider explicitly in the model the feasible possibilities.

- There is a maximum capacity (measured in square meters) for each shelter location;

- Each shelter location has a weight that can be previously computed (according to the criteria already mentioned);

- The utilization rate of each shelter must be above a value specified in advance;

- Each district must be assigned to the closest open shelter; we note that this condition is in a way, a means for considering spacial attributes of a population.

- The population of each district is assumed to be concentrated in its centroid;

- The objective is the maximization of the minimum weight of open shelters.

Before presenting an optimization model for the shelter problem we introduce the following notation to be used hereafter:

Sets:

I Set of candidate shelter locations.

Set of districts.

\section{Parameters:}

$w_{i}$ : $\quad$ weight of candidate shelter location $i \in I ; w_{i} \in[0,1]$.

$d_{j}$ : total demand of district $j \in J$ (square meters).

$q_{i}$ : $\quad$ capacity of candidate shelter location $i \in I$ (square meters).

$\ell_{i j}$ : distance between candidate shelter location $i \in I$ and district $j \in J$.

$\beta$ : $\quad$ threshold for the minimum utilization rate of a shelter.

For each $j \in J$, the distances $\ell_{i j}$ can be sorted non-decreasingly, thus providing an ordered sequence for the candidate shelter locations in terms of their distances to each district. We denote by $i_{j}(r)$ the $r$ th closest candidate shelter location to district $j \in J$ $(r=1, \ldots,|I|)$.

The decisions to be made can be represented by the following variables:

$x_{i}= \begin{cases}1 & \text { if candidate location } i \text { is chosen as a shelter area, } \\ 0 & \text { otherwise. }\end{cases}$

$(i \in I)$

$y_{i j}=\left\{\begin{array}{ll}1 & \text { if district } j \text { is assigned to shelter location } i, \\ 0 & \text { otherwise. }\end{array}(i \in I, j \in J)\right.$

$w_{\min }=$ minimum weight among the open shelters.

The following optimization model can now be proposed for the problem:

maximize $w_{\min }$,

subject to $w_{\min } \leq w_{i} x_{i}+\left(1-x_{i}\right), \quad i \in I$,

$\sum_{i \in I} y_{i j}=1, \quad j \in J$

$y_{i_{j}(1), j} \geq x_{i_{j}(1)}, \quad j \in J$, $y_{i_{j}(r), j} \geq x_{i_{j}(r)}-\sum_{s=1}^{r-1} x_{i_{j}(s)}, \quad j \in J, r=2, \ldots,|I|$,

$\sum_{j \in J} d_{j} y_{i j} \leq q_{i} x_{i}, \quad i \in I$,

$\sum_{j \in J} d_{j} y_{i j} \geq \beta q_{i} x_{i}, \quad i \in I$

$x_{i} \in\{0,1\}, \quad i \in I$,

$y_{i j} \in\{0,1\}, \quad i \in I, j \in J$.

The objective function (1) quantifies the minimum weight across the open shelters (to be maximized); Constraints (2) help defining the (linear) objective function; Constraints (3) guarantee that each district is assigned to exactly one shelter area; Constraints (4) and (5) ensure that each district is assigned to the closest open shelter area; Constraints (6) ensure that the capacity of the opened shelter areas is not exceeded; Constraints (7) define the minimum utilization rate for the opened shelters; finally, constraints (8) and (9) define the domain of the decision variables.

\subsection{A chance-constrained model}

We introduce now a stochastic version of the above problem assuming uncertainty in demands $d_{j}(j \in J)$.

Denote by $\Omega$ a finite set of scenarios such that each scenario $\omega \in \Omega$ determines a vector of demands $\left[d_{j \omega}\right]_{j \in J}$. Denote by $\gamma_{i}$ the probability that shelter $i \in I$ does not have enough capacity to handle all the demand assigned to it. We propose replacing constraints (6) by

$\mathbb{P}\left[\sum_{j \in J} d_{j} y_{i j} \leq q_{i} x_{i}\right] \geq 1-\gamma_{i}, \quad i \in I$.

Similarly, when demands are uncertain, satisfying "hard" minimum throughput constraints (7) should not be the goal. Instead, we can consider

$\mathbb{P}\left[\sum_{j \in J} d_{j} y_{i j} \geq \beta q_{i} x_{i}\right] \geq 1-\epsilon_{i}, \quad i \in I$,

which, by applying standard principles from Probability Theory leads to

$\mathbb{P}\left[\sum_{j \in J} d_{j} y_{i j} \leq \beta q_{i} x_{i}\right] \leq \epsilon_{i}, \quad i \in I$.

The values $\gamma_{i}$ and $\epsilon_{i}(i \in I)$ are typically small (e.g., 0.01 or 0.05 ) since violating the capacity and utilization rate constraints should not be highly probable. In synthesis, we propose the following chance-constrained model for the shelter site location problem under stochastic demand:

maximize (1),

subject to (2) - (5), (8) - (12).

Throughout this work, we assume that demands are independent. For many disasters this seems to be a reasonable assumption. In fact, events like hurricanes, earthquakes and flooding often have a very local effect in the sense that depending on slopes, wind, etc, the consequences may vary significantly from one village to another one that is close by. This results in demands having low correlation that we neglect in the following developments.

In a shelter site location problem a solution typically consists of several sub-districts to be served (demand points) and a small number of shelters to be installed. In the particular case of Turkey, 
if we think of a city like Istanbul, each sub-district, in fact, aggregates demand corresponding to many neighborhoods. Accordingly, a large number of neighborhoods is typically allocated to each open shelter. Therefore, we can invoke the central limit theorem to find deterministic equivalents for the probabilistic constraints (10) and (12).

For each district $j \in J$ denote by $\mu_{j}$ and $\sigma_{j}^{2}$ the expected value and variance, respectively, for its demand. The total demand to be accommodated by shelter $i \in I$ can be written as $D_{i}=\sum_{j \in J} d_{j} y_{i j}$. We know that $E\left[D_{i}\right]=\sum_{j \in J} \mu_{j} y_{i j}$. Assuming independent demands, we get $\operatorname{Var}\left[D_{i}\right]=\sum_{j \in J} \sigma_{j}^{2} y_{i j}$. When the number of terms defining $D_{i}$ is large enough, the central limit theorem assures that

$\frac{D_{i}-E\left[D_{i}\right]}{\sqrt{\operatorname{Var}\left[D_{i}\right]}} \approx N(0,1)$.

Accordingly, constraints (10) are equivalent (approximately) to

$\frac{q_{i} x_{i}-E\left[D_{i}\right]}{\sqrt{\operatorname{Var}\left[D_{i}\right]}} \geq z_{1-\gamma_{i}}, \quad i \in I$,

where $z_{1-\gamma_{i}}$ denotes the $1-\gamma_{i}$ quantile of a standardized normal distribution. A similar reasoning allow us to conclude that constraints (12) are equivalent (approximately) to

$\frac{\beta q_{i} x_{i}-E\left[D_{i}\right]}{\sqrt{\operatorname{Var}\left[D_{i}\right]}} \leq z_{\epsilon_{i}}, \quad i \in I$,

where $z_{\epsilon_{i}}$ is the the $\epsilon_{i}$ quantile of a standardized normal distribution.

Constraints (13) and (14) are non-linear due to the denominator since it includes the decision variables $y_{i j}(j \in J)$. Lin (2009) proposed handling this non-linearity by defining a new set of variables as follows:

$v_{i}=\frac{\sqrt{\sum_{j \in J} \sigma_{j}^{2} y_{i j}}}{\sqrt{\sum_{j \in J} \sigma_{j}^{2}}}, \quad i \in I$.

Obviously we have $0 \leq v_{i} \leq 1$. Furthermore, (13) and (14) together can now be replaced by

$\sum_{j \in J}\left(\frac{\mu_{j}}{\sqrt{\sum_{k \in J} \sigma_{k}^{2}}}\right) y_{i j}+z_{1-\gamma_{i}} v_{i} \leq \frac{q_{i}}{\sqrt{\sum_{k \in J} \sigma_{k}^{2}}} x_{i}, \quad i \in I$,

$\sum_{j \in J}\left(\frac{\mu_{j}}{\sqrt{\sum_{k \in J} \sigma_{k}^{2}}}\right) y_{i j}+z_{\epsilon_{i}} v_{i} \geq \frac{\beta q_{i}}{\sqrt{\sum_{k \in J} \sigma_{k}^{2}}} x_{i}, \quad i \in I$,

$v_{i}^{2}=\sum_{j \in J}\left(\frac{\sigma_{j}^{2}}{\sum_{k \in J} \sigma_{k}^{2}}\right) y_{i j}, \quad i \in I$,

$0 \leq v_{i} \leq 1, \quad i \in I$.

Accordingly, an approximate deterministic equivalent for the chance-constrained model proposed for the shelter site location problem is the following:

maximize (1),

subject to (2) - (5), (8), (9),

$$
\text { (15) }-(18) \text {. }
$$

The above model is a mixed-integer non-linear model due to the left-hand side of constraints (17). In the next section, we derive an approximation for handling these constraints.

\section{A mixed-integer linear programming approximation}

In this section, we propose replacing equalities (17) by approximating expressions. This is accomplished by considering a piecewise linear approximation of $v_{i}^{2}$ in $[0,1]$, which, in turn, can be modeled using integer variables (Beale \& Forrest, 1976; Beale \& Tomlin, 1970; Jeroslow \& Lowe, 1984).

Consider the real valued function $f\left(v_{i}\right)=v_{i}^{2}$ that maps the interval $[0,1]$ over itself. Additionally, denote by

$b_{1}=0, \quad b_{2}, \quad \ldots \quad, b_{n}-1, \quad b_{n}=1$

a set of breakpoints inducing a partition of $[0,1]$.

For every $i \in I$, taking $v_{i} \in[0,1]$, there is $m \in\{0, \ldots, n-1\}$ such that $v_{i} \in\left[b_{m}, b_{m+1}\right]$, i.e., $v_{i}$ can be written as a linear convex combination of $b_{m}$ and $b_{m+1}$ :

$v_{i}=\lambda_{i m} b_{m}+\lambda_{i m+1} b_{m+1}$

with $\lambda_{i m}+\lambda_{i m+1}=1$ and $\lambda_{i m}, \lambda_{i m+1} \geq 0$.

For every $m \in\{0, \ldots, n-1\}$,

$\overline{f_{m}}(v)=\left(b_{m}+b_{m+1}\right) v-b_{m} b_{m+1}$

is the line segment connecting points $\left(b_{m}, b_{m}^{2}\right)$ and $\left(b_{m+1}, b_{m+1}^{2}\right)$. Finally, $v_{i}^{2}$ can be approximated by $\bar{f}_{m}\left(v_{i}\right)=\lambda_{i m} b_{m}^{2}+\lambda_{i m+1} b_{m+1}^{2}$.

The previous analysis shows that for every $i \in I, v_{i}$ can be written as $v_{i}=\sum_{m=1}^{n} \lambda_{i m} b_{m}$, and $v_{i}^{2}$ can be approximated (from above-the function being approximated is convex) by $\sum_{m=1}^{n} \lambda_{i m} b_{m}^{2}$, given that $\lambda_{i m}$ are non-negative values satisfying

$\sum_{m=1}^{n} \lambda_{i m}=1$,

$\lambda_{i m} \leq t_{i m}, \quad m=1, \ldots, n$,

$\sum_{m=1}^{n} t_{i m} \leq 2$

$t_{i m}+t_{i m^{\prime}} \leq 1, \quad m=1, \ldots, n-2 ; m^{\prime}=m+2, \ldots, n$,

$t_{i m} \in\{0,1\}, \quad m=1, \ldots, n$.

For each $i \in I$, the binary variables $t_{i m}(m=1, \ldots, n)$ ensure that at most two breakpoints are used to define one value of the piecewise linear function and if two of them are used then they must be adjacent. In other words, variables $\left\{t_{i 1}, \ldots, t_{i n}\right\}$ define a special ordered set of type 2 (SOS2) (see, e.g., Beale \& Forrest (1976)).

The full (approximate) deterministic equivalent model to be solved now will be denoted by $(\mathrm{P})$ and is the following:

maximize $W_{\min }$,

subject to $W_{\min } \leq w_{i} x_{i}+\left(1-x_{i}\right), \quad i \in I$,

$\sum_{i \in I} y_{i j}=1, \quad j \in J$,

$y_{i_{j}(1), j} \geq x_{i_{j}(1)}, \quad j \in J$,

$y_{i_{j}(r), j} \geq x_{i_{j}(r)}-\sum_{s=1}^{r-1} x_{i_{j}(s)}, \quad j \in J, r=2, \ldots,|I|$,

$\sum_{j \in J}\left(\frac{\mu_{j}}{\sqrt{\sum_{k \in J} \sigma_{k}^{2}}}\right) y_{i j}+z_{1-\gamma_{i}} \sum_{m=1}^{n} \lambda_{i m} b_{m} \leq \frac{q_{i}}{\sqrt{\sum_{k \in J} \sigma_{j}^{2}}} x_{i}, \quad i \in I$,

$\sum_{j \in J}\left(\frac{\mu_{j}}{\sqrt{\sum_{k \in J} \sigma_{k}^{2}}}\right) y_{i j}+z_{\epsilon_{i}} \sum_{m=1}^{n} \lambda_{i m} b_{m} \geq \frac{\beta q_{i}}{\sqrt{\sum_{k \in J} \sigma_{j}^{2}}} x_{i}, \quad i \in I$, 
$\sum_{j \in J}\left(\frac{\sigma_{j}^{2}}{\sum_{k \in J} \sigma_{k}^{2}}\right) y_{i j}=\sum_{m=1}^{n} \lambda_{i m} b_{m}^{2}, \quad i \in I$,

$\sum_{m=1}^{n} \lambda_{i m}=1, \quad i \in I$,

$\lambda_{\text {im }} \leq t_{i m}, \quad i \in I, m=1, \ldots, n$,

$\sum_{m=1}^{n} t_{i m} \leq 2, \quad i \in I$,

$t_{i m}+t_{i m^{\prime}} \leq 1, \quad i \in I, m=1, \ldots, n-2 ; m^{\prime}=m+2, \ldots, n$,

$x_{i} \in\{0,1\}, \quad i \in I$,

$y_{i j} \in\{0,1\}, \quad i \in I, j \in J$,

$t_{i m} \in\{0,1\}, \quad i \in I, m=1, \ldots, n$,

$\lambda_{\text {im }} \geq 0, \quad i \in I, m=1, \ldots, n$.

Constraints (26)-(28) are the SOS2 constraints. We also note that instead of $(25)$ we can consider

$\sum_{m=1}^{n} \lambda_{i m}=x_{i}, \quad i \in I$.

In fact, for some $i \in I$, if $x_{i}=1$ then we have the original constraints; otherwise, we have $\lambda_{i 1}=\ldots=\lambda_{\text {in }}=0$ which, by (24) together with (9) renders $y_{i j}=0, j \in J$ and thus constraints (15) and (16) become trivial inequalities not relevant the problem (as it should be the case if shelter $i$ is not selected to open). The new right hand side for constraints (25) strengthens the linear programming relaxation of model $(\mathrm{P})$.

The previous model makes use of the closest assignment constraints (4) and (5) also considered by Kilcl et al. (2015). These constraints were introduced into the literature by Rojeski and ReVelle (1970). They are referred to as the $R R$ closest assignment constraints (Espejo, Marìn, \& Rodrìguez-Chìa 2012). If larger instances of the problem are to be solved, other possibilities can be considered. In this paper given that we assume all distances known we can consider the following alternative:

$\sum_{s=r+1}^{|I|} y_{i_{j}(s), j}+x_{i_{j}(r)} \leq 1, \quad j \in J, r=1, \ldots,|I-1|$.

Constraints (31) were first proposed by Wagner and Falkson (1975). As shown by Espejo et al. (2012), these constraints dominate the $R R$ constraints. In the next section we show results concerning their use in the context of our problem.

\section{Computational experiments}

In this section, we use the approximation model derived in Section 4 for assessing the relevance of considering chance constraints in the stochastic shelter site location problem.

We start by presenting results using the data reported by Kilc1 et al. (2015). This is important to test the relevance of considering a stochastic approach instead of a deterministic one. Afterwards, we use the approximate model for a large data set that includes the whole Anatolian side of Istanbul.

The deterministic model and the mixed-integer approximation were coded in Optimization Programming Language (OPL) and were solved using IBM CPLEX 12.6.1. All tests were run on a Linux
OS with four AMD Opteron Interlagos 6282SE 16 Core 2.6 gigahertz 16 megabytes L3 cache server processors with 96 gigabytes of RAM.

For both data sets (the one introduced by Kilc1 et al. (2015) and the large-scale one introduced in the current work), 51 breakpoints were used in the piecewise linear approximations required to formulate model (P). Although our experience with some specific running configurations shows that a much lower number of breakpoints renders approximations with a negligible error, we decided to keep a large value in order to be safe in terms of the accuracy of the presented results. Accordingly, the interval $[0,1]$ was always partitioned into 50 sub-intervals.

In all tests reported in this section, constraints $\left(25^{\prime}\right)$ were used instead of (25).

Before presenting the results obtained namely, the optimal solutions for the instances studied, we would like to point out that for a specific solution we can develop measures that allow us to understand features such as fairness, accessibility, efficiency, and equity. These are important aspects when it comes to analyzing a solution to our problem.

Regarding fairness we note that our objective-maximization of the minimum shelter weight-targets the least-advantaged districts covered in the problem. In other words, by considering that objective function, we are targeting fairness for the least advantaged disaster victims. Additionally, for all the specific solutions that will be presented next, additional measures were computed, namely: the average walking distance and the maximum walking distance. The average walking distance provides a measure of accessibility. In turn, the maximum travel distance can be looked at as an efficiency measure since it gives an indication of how far from the closest shelter is the population group that needs to walk the most. The lower this value the more efficient the system can be looked at; in fact, a small maximum distance tells us that all the demand points have a shelter close by. Another efficiency measure concerns the utilization rates of the shelters. The higher the average utilization rate the more efficiently the shelters are being used. Finally, regarding equity, we can compare the maximum travel distance with the average travel distance. The closer these values are to each other the more fairness the system exhibits. Likewise, we can compare the highest shelter utilization rate with the average. The farther away these values are from each other the more we find some shelters less populated than others, giving an indication that in the former case, accommodated populations have more utility than in the latter.

\subsection{Computational results for the Kartal district}

Kartal is one of the 39 districts of Istanbul with an area of 38.54 square kilometers and with approximately 425,000 inhabitants. In Kartal, there are 25 potential shelter locations, whose weights range from 0.674 to 0.982 with an average of 0.827 and a standard deviation of 0.097. 20 demand points can be considered, each one corresponding to the centroid of one sub-district of Kartal and whose number of inhabitants is known. This data set was constructed by Unal (2010) as part of his Ph.D. thesis where he conducted extensive surveys and analysis with experts.

The above data would be used later on by Kilc1 et al. (2015) who considered a so-called Percent Affected Ratio (PAR) of 0.125 . This value indicates that $12.5 \%$ of the population would need to stay in a shelter after an earthquake. However, in the event of a disaster, hardly will this parameter be known in advance; as discussed before, it may depend on different aspects. This means that when shelter locations are being decided, varying demand should not be neglected. We capture such variability via PAR. In particular, we start by considering three different patterns, all centered in the original value considered by Kilc1 et al. (2015): 

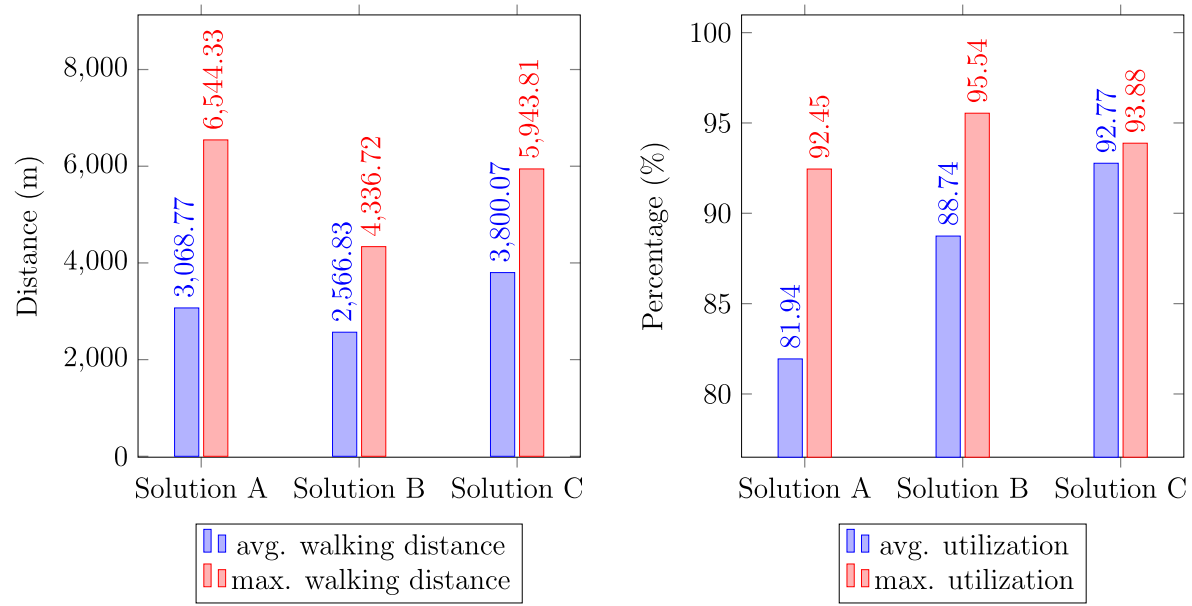

Fig. 1. Comparison of deterministic solutions with respect to additional measures.

Table 2

Solutions obtained using the deterministic models (1)-(9)

\begin{tabular}{|c|c|c|c|c|c|}
\hline \multirow{2}{*}{\multicolumn{2}{|c|}{$\frac{\text { (a) } \beta=0.70}{\text { Solution } \mathrm{A}}$}} & \multirow{2}{*}{\multicolumn{2}{|c|}{$\begin{array}{l}\text { (b) } \beta=0.80 \\
\text { Solution } \mathrm{B}\end{array}$}} & \multirow{2}{*}{\multicolumn{2}{|c|}{$\begin{array}{l}\beta=0.90 \\
\text { Solution C } \\
\end{array}$}} \\
\hline & & & & & \\
\hline Objective value: & 0.847 & Objective value: & 0.803 & Objective value: & 0.739 \\
\hline Open shelters: & $10-19-25$ & Open shelters: & $4-10-14$ & Open shelters: & $14-23$ \\
\hline
\end{tabular}

- Pattern 1 - high variability: PAR $=0.125 \times U[0.85,1.15]$

- Pattern 2 - moderate variability: $P A R=0.125 \times U[0.90,1.10]$

- Pattern 3 - low variability: $P A R=0.125 \times U[0.95,1.05]$

$U[a, b]$ denotes a random number generated according to a continuous uniform distribution in the interval $[a, b]$. For each pattern above and for each sub-district $j \in J$, ten values were generated for $P A R$. Each one was then multiplied by the number of inhabitants associated with that demand point and also by 3.5 square meters (recall that in our problem the demand is measured in square meters). Accordingly, for each PAR pattern used a sample was obtained containing 10 possible values for the demand of location $j \in J$. From each such sample it was possible to estimate $\mu_{j}$ and $\sigma_{j}^{2}$ by means of the corresponding sample values. We call demand pattern to the sample of 10 values for the demand obtained according to one of the above PAR generation schemes, i.e., we have demand pattern 1, demand pattern 2, and demand pattern 3 .

We also note that the expected value for PAR is always 0.125 which means that we are keeping the expected values of this parameter around that value. This is a way for understanding the effect of the variability of demand in the solutions keeping the expected demands equal to the values considered by Kilc1 et al. (2015).

In the following analysis for Kartal district we never refer to CPU time since model $(\mathrm{P})$ was always solved within a few seconds that we consider not significant enough to report in this paper.

\subsubsection{Results using the deterministic model}

Using the data introduced by Kilc1 et al. (2015), i.e., considering the single value for the demand induced by $P A R=0.125$, we solved the deterministic model (1)-(9). We run the model using three different thresholds in terms of the minimum utilization rate of a shelter: $70 \%, 80 \%$ and $90 \%$ (i.e., $\beta=0.7,0.8,0.9$, respectively). The results are presented in Table 2. The details of each solution in terms of accessibility, efficiency and equity measures are shown on Fig. 1. For instance, solution A was obtained when $\beta$ was set equal to 0.7 . This led to the selection of shelters 10,19 and 25 and to an optimal objective value (minimum weight of the open shelters) of 0.847 .

As we can observe in Table 2, an increase in $\beta$ corresponds to a decrease in the optimal value of the problem, i.e., we get a decrease in the solution fairness. This is not surprising since the stricter we are in terms of the minimum utilization rate the more flexible we must be in terms of considering alternative shelters (thus with a lower weight) and the less fair the solution may become.

Observing these solutions in more depth as depicted in Fig. 1, we conclude that for solution $C$ the average traveled distance is the largest, indicating a smaller level of accessibility when compared with other solutions. On the other hand, the maximum traveled distance is achieved in solution A indicating that this solution seems not to be so efficient as the other two. Interestingly, when we compare the maximum travel distance with the average, we obtain the smallest difference in solution $B$ indicating a higher equity level. Moreover, for solution $\mathrm{A}$, we observe that number of disaster victims covering the maximum travel distance is $8.7 \%$ of total number of disaster victims whereas this proportion is $5.2 \%$ and $2.4 \%$ for solution B and solution C, respectively. This points out another important aspect of analysis: the level of maximum travel distance measure based on the percentage of people serviced at that level. Naturally, the lower this percentage is, the lower influence maximum travel distance measure on the quality of the solution has.

Another interpretation of the results can be based on the average and maximum utilization rates of the solutions. As it is expected, an increase in $\beta$ results in an increase in the average utilization rates as they are bounded from below by this value. We also observe that the equity between open shelters increases towards solution $\mathrm{C}$ as the difference between two aforementioned measures are decreasing, which indicates that the utilizations of open shelters are closer to each other.

\subsubsection{Results using the approximate model $(P)$}

The next step in our computational experiments was to solve the approximate model $P$ using different values of $\gamma_{i}$ and $\epsilon_{i}, i \in I$. Since we considered the same values for all potential shelter site locations $i \in I$, hereafter we denote those probabilities simply by $\epsilon$ and $\gamma$.

For both probabilities we considered the values $0.01,0.0275$, $0.05,0.075$, and 0.1. This resulted in the values $0.990,0.975$. $0.950,0.925,0.900$, respectively for $1-\gamma_{i}$, which can be looked at as a "shelter coverage level". The combinations of the different values for $1-\gamma$ and $\epsilon$ yields 25 different instances. In turn, each 
Table 3

Results for $\beta=0.70$ and low variability in demand.

\begin{tabular}{llllll}
\hline (a) Optimal solution. & \multicolumn{5}{l}{} \\
\hline & $\varepsilon_{0.010}$ & $\epsilon_{0.025}$ & $\epsilon_{0.050}$ & $\epsilon_{0.075}$ & $\epsilon_{0.100}$ \\
\hline$\gamma_{0.010}$ & $A$ & $A$ & $A$ & $A$ & $A$ \\
$\gamma_{0.025}$ & $A$ & $A$ & $A$ & $A$ \\
$\gamma_{0.050}$ & $A$ & $A$ & $A$ & $A$ & $A$ \\
$\gamma_{0.075}$ & $A$ & $A$ & $A$ & $A$ & $A$ \\
$\gamma_{0.100}$ & $A$ & $A$ & $A$ & $A$ & $A$ \\
(b) Solution A - capacity used (\%). & & & & \\
\hline Open shelters & Maximum (\%) & Average (\%) & Minimum (\%) \\
\hline 10 & 97 & 92 & & 89 \\
19 & 77 & 73 & & 77 \\
25 & 84 & 80 & & \\
\hline
\end{tabular}

Table 4

New solutions emerging when the (approximated) chance-constrained model is used.

\begin{tabular}{llllll}
\hline Solution D & \multicolumn{3}{l}{ Solution F } \\
\hline Objective value: & 0.809 & Objective value: & 0.801 & Objective value: & 0.801 \\
Open shelters: & $13-18-19-25$ & Open shelters: & $8-9-12-25$ & Open shelters: & $4-8-9-12$ \\
\hline
\end{tabular}

Table 5

Results for $\beta=0.70$ and moderate variability in demand.

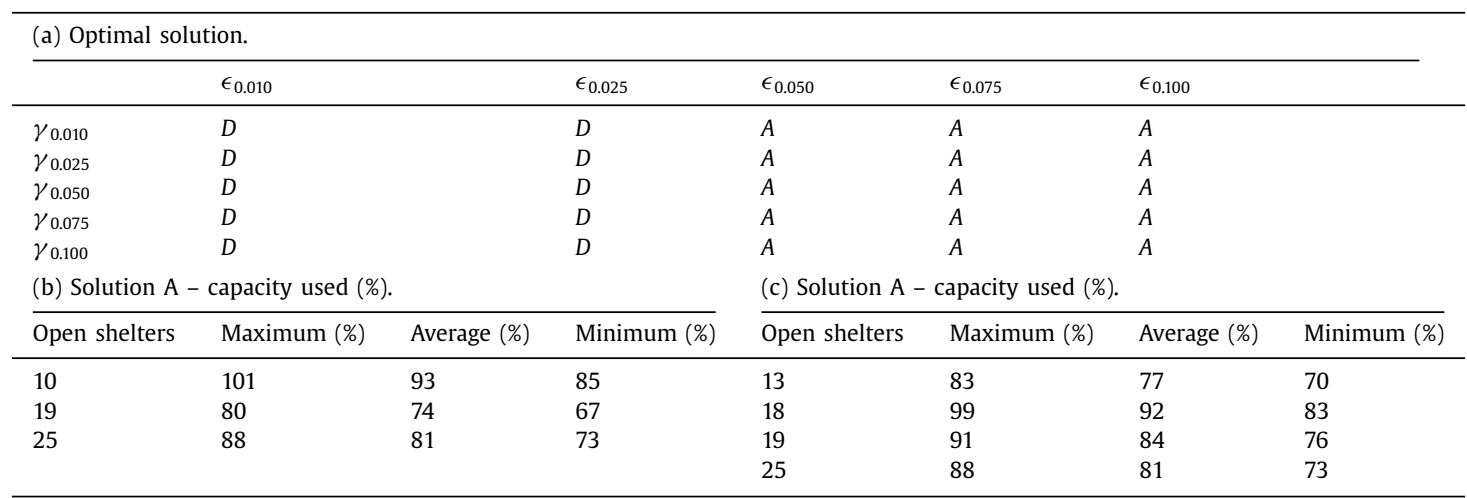

combination can be considered for each of the three demand patterns considered. This will be reported next. We perform a full analysis for $\beta=0.7,0.8,0.9$.

Results for $\boldsymbol{\beta}=0.7$.

The results obtained for $\beta=0.7$ can be found in Tables 3, 5, and 6.

We can observe that Table 3 a is filled with solution A-the deterministic solution obtained for $\beta=0.7$. For this same value of $\beta$, when the demand variability increases, new solutions emerge namely, those depicted in Table 4 . In particular, the dominance of solution A decreases as we can observe in the solutions depicted in Table 5a (for medium demand variability) and in Table 6a (for higher demand variability).

It interesting to observe from Fig. 2 that when the chanceconstrained model was considered, the quality measures we have been considering (accessibility, efficiency, and equity) improve without a deterioration in our fairness objective. This is a clear indication that a model capturing uncertainty can better hedge against it yielding a solution that overall can be considered better than a solution ignoring uncertainty.

For moderate demand variability we can observe in Table 5 a that a new solution, solution $\mathrm{D}$, emerges as an optimal solution to model (P).

This solution has a lower objective value than solution $A$ but the open shelters can handle the $\epsilon$-strictness better than in solution A. From the utilization rates (Table $5 \mathrm{~b}$ and $5 \mathrm{c}$ ) one can conclude that solution D has neither values greater than $100 \%$ nor lower than $70 \%$ whereas solution A can be out of these boundaries in the extreme cases.

Finally, also for $\beta=0.7$ we analyze the results when demand exhibits the highest variability (Table 6). A new solution emerges now (solution F). Nevertheless, in Table 6a we can see that solutions $\mathrm{E}$ and $\mathrm{F}$ are alternative optima for many combinations of $\epsilon$ and $\gamma$. In fact, they have the same objective value of 0.801 . However, from Fig. 2, it can be seen that despite having the same maximum walking distance value, solution $\mathrm{F}$ is superior in terms of accessibility since it renders a better average walking distance. Similarly, both solutions $\mathrm{E}$ and $\mathrm{F}$ have the same maximum utilization rates, whereas solution $\mathrm{F}$ has a higher average utilization rate, which indicates that its fairness is relatively higher. On the other hand, solution A does not seem to cope well with high variability; it is only valid on the left-down corner (most loose part) of $\epsilon$ vs. $\gamma$ table.

Results for $\boldsymbol{\beta}=0.8$.

The results obtained when $\beta$ was set to 0.8 can be found inTables 7-9 for the different demand patterns. In these tables, we can see a behavior similar to that observed for $\beta=0.7$. Again, the deterministic solution-now solution $\mathrm{B}$-dominates all through the optimal results of model $(\mathrm{P})$ when the lowest variability is considered for the demand (Table 7a). With an increase in the demand variability, solution B ceases to be the optimal one. Moreover, the problem starts to become infeasible for some combinations of $\gamma$ 
Table 6

Results for $\beta=0.70$ and high variability in demand.

(a) Optimal solution.

\begin{tabular}{|c|c|c|c|c|c|}
\hline & $\epsilon_{0.010}$ & $\epsilon_{0.025}$ & $\epsilon_{0.050}$ & $\epsilon_{0.075}$ & $\epsilon_{0.100}$ \\
\hline$\gamma_{0.010}$ & $E$ or $F$ & $E$ or $F$ & $E$ or $F$ & $E$ or $F$ & $E$ or $F$ \\
\hline$\gamma_{0.025}$ & $E$ or $F$ & $E$ or $F$ & $E$ or $F$ & $E$ or $F$ & $E$ or $F$ \\
\hline$\gamma_{0.050}$ & $E$ or $F$ & $E$ or $F$ & $E$ or $F$ & $E$ or $F$ & $A$ \\
\hline$\gamma_{0.075}$ & $E$ or $F$ & $E$ or $F$ & $E$ or $F$ & $E$ or $F$ & $A$ \\
\hline$\gamma_{0.100}$ & $E$ or $F$ & $D$ & $D$ & $D$ & $A$ \\
\hline
\end{tabular}

(b) Solution A - capacity used (\%).

(c) Solution D - capacity used (\%).

\begin{tabular}{|c|c|c|c|c|c|c|c|}
\hline Open shelters & Maximum (\%) & Average (\%) & Minimum (\%) & Open shelters & Maximum (\%) & Average (\%) & Minimum (\%) \\
\hline 10 & 105 & 94 & 80 & 13 & 87 & 78 & 66 \\
\hline 19 & 84 & 75 & 63 & 18 & 104 & 93 & 78 \\
\hline 25 & 91 & 82 & 69 & 19 & 95 & 85 & 72 \\
\hline & & & & 25 & 91 & 82 & 69 \\
\hline
\end{tabular}

(d) Solution E - capacity used (\%).

(e) Solution F - capacity used (\%).

\begin{tabular}{|c|c|c|c|c|c|c|c|}
\hline Open shelters & Maximum (\%) & Average (\%) & Minimum (\%) & Open shelters & Maximum (\%) & Average (\%) & Minimum (\%) \\
\hline 8 & 98 & 88 & 74 & 4 & 98 & 88 & 74 \\
\hline 9 & 97 & 87 & 74 & 8 & 97 & 87 & 74 \\
\hline 12 & 92 & 82 & 69 & 9 & 99 & 88 & 72 \\
\hline 25 & 91 & 82 & 69 & 12 & 97 & 77 & 73 \\
\hline
\end{tabular}

Table 7

Results for $\beta=0.80$ and low variability in demand.

\begin{tabular}{|c|c|c|c|c|c|}
\hline \multicolumn{5}{|c|}{ (a) Optimal solution. } & \multirow[b]{2}{*}{$\epsilon_{0.100}$} \\
\hline & $\epsilon_{0.010}$ & $\epsilon_{0.025}$ & $\epsilon_{0.050}$ & $\epsilon_{0.075}$ & \\
\hline$\gamma_{0.010}$ & $B$ & $B$ & $B$ & $B$ & $B$ \\
\hline$\gamma_{0.025}$ & $B$ & $B$ & $B$ & $B$ & $B$ \\
\hline$\gamma_{0.050}$ & $B$ & $B$ & $B$ & $B$ & $B$ \\
\hline$\gamma_{0.075}$ & $B$ & $B$ & $B$ & $B$ & $B$ \\
\hline$\gamma_{0.100}$ & $B$ & $B$ & $B$ & $B$ & $B$ \\
\hline \multicolumn{5}{|c|}{ (b) Solution B - capacity used (\%). } & \\
\hline & Open shelters & Maximum (\%) & Average (\%) & Minimum (\%) & \\
\hline & 4 & 89 & 85 & 82 & \\
\hline & 10 & 90 & 86 & 83 & \\
\hline & 14 & 100 & 96 & 92 & \\
\hline
\end{tabular}

Table 8

Results for $\beta=0.80$ and moderate variability in demand.

\begin{tabular}{llllll}
\hline \multicolumn{2}{l}{ (a) Optimal solution. } & & & & \\
\hline & $\epsilon_{0.010}$ & $\epsilon_{0.025}$ & $\epsilon_{0.050}$ & $\epsilon_{0.075}$ & $\epsilon_{0.100}$ \\
\hline$\gamma_{0.010}$ & Inf & Inf & Inf & Inf & Inf \\
$\gamma_{0.025}$ & Inf & Inf & Inf & Inf & Inf \\
$\gamma_{0.050}$ & Inf & Inf & Inf & Inf & Inf \\
$\gamma_{0.075}$ & $C$ & $C$ & $C$ & $C$ & $C$ \\
$\gamma_{0.100}$ & $C$ & $C$ & $C$ & & Minimum (\%) \\
(b) Solution C & - capacity used (\%). & & & 86 & \\
\hline \multicolumn{7}{c}{ Open shelters } & Maximum (\%) & Average (\%) & \\
\hline
\end{tabular}

and $\epsilon$. In particular, a few extra computations with the data led us to conclude that for the highest variability in the demand, feasible solutions start to be obtained only if we considered values of $\gamma$ larger than 0.23 .

In Table $7 \mathrm{~b}$, we observe that if solution B was implemented, the lower and upper limits in terms of utilization rate of the open shelters are between 82 and $100 \%$. In other words, for the probabilities considered, the solution is always feasible. However, when we move to Table 8 we observe that if we implemented solution $\mathrm{C}$, in some cases, the utilization rates are slightly higher than the $100 \%$. In other words, we are observing that in some cases, solution $C$ is not a feasible solution (as no solution is-see the first three rows in Table $8 \mathrm{a}$ ). Therefore, we conclude that when $\beta=0.8$, for some demand patterns the values selected for $\gamma$ and $\epsilon$ were too strict. Similarly, for Table 9, we present utilization rates for solution $\mathrm{C}$ if it was implemented which allow us to conclude why the solution is never feasible: the utilization rate shows maximum values significantly above $100 \%$ and minimum values below $80 \%$. As stated in Table 9a no feasible solution was found for the combinations of $\gamma$ and $\epsilon$ analyzed. Again, this indicates that for $\beta=0.8$ the values selected for $\gamma$ and $\epsilon$ were too strict.

Results for $\boldsymbol{\beta}=0.9$.

The results obtained for $\beta=0.9$ are depicted in Tables $10-12$. Like for $\beta=0.8$ we observe that for some demand patterns, the 


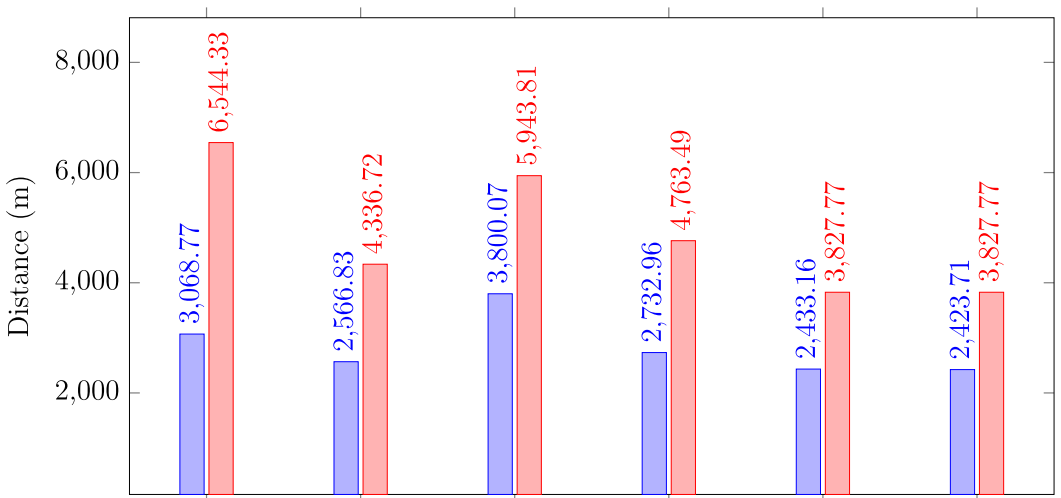

Solution A Solution B Solution C Solution D Solution E Solution F

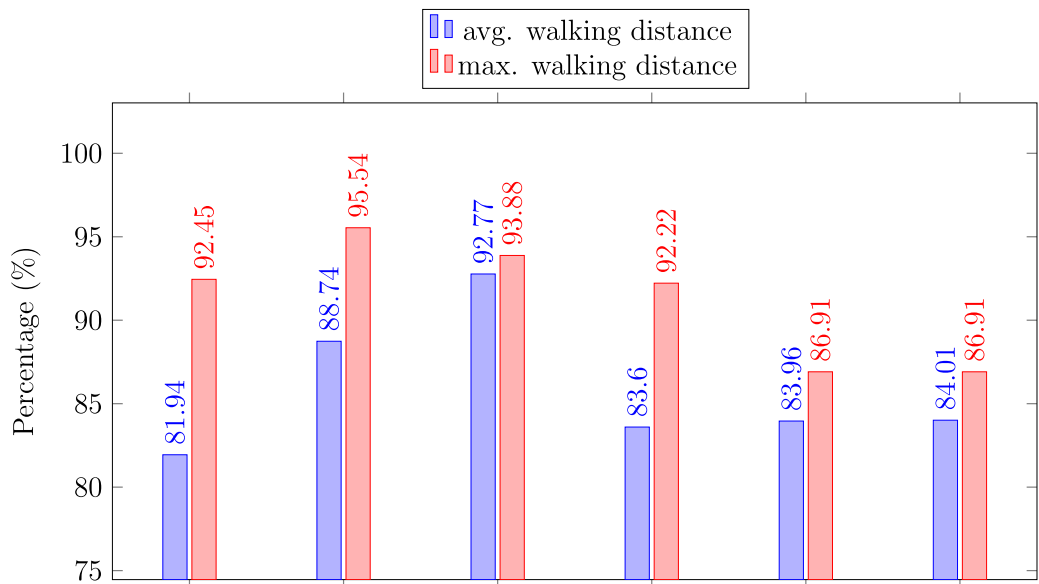

Solution A Solution B Solution C Solution D Solution E Solution F

\begin{tabular}{l}
\hline$\square$ avg. utilization \\
$\square \square \max$. utilization
\end{tabular}

Fig. 2. Comparison of new solutions with respect to additional measures.

Table 9

Results for $\beta=0.80$ and high variability in demand.

\begin{tabular}{|c|c|c|c|c|c|}
\hline \multicolumn{5}{|c|}{ (a) Optimal solution. } & \multirow[b]{2}{*}{$\epsilon_{0.100}$} \\
\hline & $\epsilon_{0.010}$ & $\epsilon_{0.025}$ & $\epsilon_{0.050}$ & $\epsilon_{0.075}$ & \\
\hline$\gamma_{0.010}$ & Inf & Inf & Inf & Inf & Inf \\
\hline$\gamma_{0.025}$ & $\operatorname{Inf}$ & $\operatorname{Inf}$ & Inf & $\operatorname{Inf}$ & $\operatorname{Inf}$ \\
\hline$\gamma_{0.050}$ & $\operatorname{Inf}$ & $\operatorname{Inf}$ & Inf & Inf & Inf \\
\hline$\gamma_{0.075}$ & Inf & Inf & Inf & Inf & Inf \\
\hline$\gamma_{0.100}$ & $\operatorname{Inf}$ & $\operatorname{Inf}$ & $\operatorname{Inf}$ & $\operatorname{Inf}$ & $\operatorname{Inf}$ \\
\hline \multicolumn{6}{|c|}{ (b) (Infeasible) solution C - capacity used (\%) if the solution was implemented. } \\
\hline & Open shelters & Maximum (\%) & Average (\%) & Minimum (\%) & \\
\hline & 14 & 103 & 95 & 86 & \\
\hline & 23 & 101 & 93 & 84 & \\
\hline
\end{tabular}

Table 10

Results for $\beta=0.90$ and low variability in demand.

\begin{tabular}{llllll}
\hline \multicolumn{2}{l}{ (a) Optimal solution. } & & & & \\
\hline & $\epsilon_{0.010}$ & $\epsilon_{0.025}$ & $\epsilon_{0.050}$ & $\epsilon_{0.075}$ & \\
\hline$\gamma_{0.010}$ & Inf & Inf & $C$ & $C$ & $C$ \\
$\gamma_{0.025}$ & Inf & Inf & $C$ & $C$ & $C$ \\
$\gamma_{0.050}$ & Inf & Inf & $C$ & $C$ & $C$ \\
$\gamma_{0.075}$ & Inf & Inf & $C$ & $C$ & $C$ \\
$\gamma_{0.100}$ & Inf & Inf & $C$ & $C$ & C \\
(b) Solution C & - capacity used (\%). & & & & Minimum (\%) \\
\hline \multicolumn{7}{c}{ Open shelters } & Maximum (\%) & Average (\%) & 91 \\
\hline
\end{tabular}


Table 11

Results for $\beta=0.90$ and low variability in demand.

\begin{tabular}{llllll}
\hline \multicolumn{2}{l}{ Optimal solution. } & \multicolumn{3}{l}{} \\
\hline & $\epsilon_{0.010}$ & $\epsilon_{0.025}$ & $\epsilon_{0.050}$ & $\epsilon_{0.075}$ & $\epsilon_{0.100}$ \\
\hline$\gamma_{0.010}$ & Inf & Inf & Inf & Inf & Inf \\
$\gamma_{0.025}$ & Inf & Inf & Inf & Inf & Inf \\
$\gamma_{0.050}$ & Inf & Inf & Inf & Inf & $C$ \\
$\gamma_{0.075}$ & Inf & Inf & Inf & Inf & Inf \\
$\gamma_{0.100}$ & Inf & Inf & Inf & & Minimum (\%) \\
(b) Solution C & - capacity used (\%). & & & 86 & \\
\hline \multicolumn{7}{c}{ Open shelters } & Maximum (\%) & Average (\%) & \\
\hline
\end{tabular}

Table 12

Results for $\beta=0.90$ and high variability in demand.

\begin{tabular}{llllll}
\hline & $\epsilon_{0.010}$ & $\epsilon_{0.025}$ & $\epsilon_{0.050}$ & $\epsilon_{0.075}$ & $\epsilon_{0.100}$ \\
\hline$\gamma_{0.010}$ & Inf & Inf & Inf & Inf & Inf \\
$\gamma_{0.025}$ & Inf & Inf & Inf & Inf & Inf \\
$\gamma_{0.050}$ & Inf & Inf & Inf & Inf & Inf \\
$\gamma_{0.075}$ & Inf & Inf & Inf & Inf & Inf \\
$\gamma_{0.100}$ & Inf & Inf & Inf & Inf & Inf \\
\hline
\end{tabular}

values for $\gamma$ and $\epsilon$ were too strict to allow obtaining a feasible solution. This means that if we impose a minimum utilization shelter rate of $90 \%$ then we must increase the probabilities $\gamma$ and/or $\epsilon$.

Model (P) was developed considering an important assumption: the validity of the central limit theorem for the instance at hand. It should be noted that that the number of sub-districts assigned to a shelter site is generally less than 30 . Nevertheless, the demand of each sub-district is itself the result of the aggregation (i.e. the sum) of the demand of many neighborhoods. Accordingly, when we are adding the demand of one or several sub-districts we are certainly considering a number of neighborhoods clearly above 30 thus, we are on the safe side when invoking the CLT.

\subsection{Results for large-scale data}

In order to evaluate the behavior of model (P) when large-scale data is used, we considered the whole Anatolian Side of Istanbul. Such data set contains 270 candidate shelter locations and 230 districts (demand points). Overall we have a 500-node instance. All the corresponding information has been gathered for this study (population, potential shelters weights, etc.) and it can be made available upon a request to the authors.

In these tests, we assumed $P A R=0.125$; we considered demand pattern 1-lower variability in demand; we set $\gamma=\epsilon=0.10$. Regarding $\beta$ we set it to 0.00 , i.e., we considered full flexibility in terms of the minimum utilization rate of the selected shelters. This was a way to look into the importance of such threshold.

Using model $(\mathrm{P})$, we were able to obtain a solution in 43 hours and 48 minutes. The selected shelters as well as their weights and their utilization rates are presented in Table 13. Such solution calls for opening 26 shelters whose minimum weight (objective function value) corresponds to the weight of shelter 46 and is equal to 0.781146 . Regarding the utilization rates, we observe that the minimum value is $38.54 \%$. From this Table we computed the average which is equal to $71.75 \%$.

Since the CPU time turned out to be excessively high, we explored the possibility of using constraints (31) instead of (4) and (5). By doing so, we were able to obtain the same solution detailed in Table 13 but taking only 4 hours and 24 minutes, which represents a $89.9 \%$ improvement in the CPU time.
Table 13

Solution information for the Anatolian side of Istanbul.

\begin{tabular}{llllll}
\hline Shelter & Weight & $\begin{array}{l}\text { Utilization } \\
\text { rate (\%) }\end{array}$ & Shelter & Weight & $\begin{array}{l}\text { Utilization } \\
\text { rate (\%) }\end{array}$ \\
\hline 5 & 0.788021 & 98.39 & 111 & 0.866857 & 71.98 \\
8 & 0.844608 & 89.99 & 136 & 0.876425 & 65.83 \\
15 & 0.843545 & 64.25 & 166 & 0.855216 & 91.04 \\
24 & 0.793249 & 42.26 & 168 & 0.796147 & 93.85 \\
33 & 0.850611 & 51.61 & 169 & 0.805162 & 38.54 \\
41 & 0.876523 & 96.25 & 171 & 0.893454 & 55.35 \\
46 & $\mathbf{0 . 7 8 1 1 4 6}$ & 47.72 & 175 & 0.866260 & 56.30 \\
49 & 0.873054 & 97.97 & 195 & 0.820383 & 86.46 \\
50 & 0.813283 & 98.07 & 200 & 0.810284 & 65.51 \\
72 & 0.819059 & 83.32 & 204 & 0.791751 & 86.46 \\
81 & 0.837859 & 90.66 & 210 & 0.877855 & 50.57 \\
91 & 0.800685 & 41.28 & 216 & 0.790012 & 54.10 \\
95 & 0.891362 & 90.72 & 220 & 0.800323 & 56.94 \\
\hline
\end{tabular}

Table 14

CPU times for the two enhancements studied.

\begin{tabular}{lll}
\hline $\begin{array}{l}\text { Closest assignment } \\
\text { constraints }\end{array}$ & $\begin{array}{l}\text { CPU times using } \\
(26)-(29)\end{array}$ & $\begin{array}{l}\text { CPU times defining the } \\
\text { SOS2's }\end{array}$ \\
\hline (4) and (5) & $\begin{array}{l}43 \text { hours and 48 } \\
\text { minutes }\end{array}$ & $\begin{array}{l}20 \text { hours and } 21 \\
\text { minutes }\end{array}$ \\
& $\begin{array}{l}4 \text { hours and 24 hours and 2 minutes } \\
\text { minutes }\end{array}$ & \\
\hline
\end{tabular}

As mentioned when introducing constraints (19)-(23), for each $i \in I,\left\{t_{i 1}, \ldots, t_{i n}\right\}$ defines a special ordered set of type 2 (SOS2). This feature can be explicitly set in CPLEX, thus avoiding having to consider constraints (26)-(29) in model (P) explicitly.

Table 14 summarizes the CPU time comparisons considering this alternative implementation as well as considering the closest assignment constraints (31) instead of (4) and (5).

We can observe that defining the SOS2 explicitly and using (31) as the closest assignment constraints renders a prominent combination to use for obtaining the best CPU time performance.

The results we obtained are encouraging in the sense that when uncertainty exists in a shelter site location problem (and it is likely that it indeed exists), considering a modeling framework such as the one we proposed in this paper, may render better solutions then if a deterministic model was considered. By better solutions we do not mean that they are better for a particular scenario or realization of the uncertainty. That is not the case. In fact, if we knew the exact demand for sheltering, then our model would not be necessary. In that case, we should directly consider a single-scenario (deterministic) model and find the optimal solution for it. What our results show is that under uncertainty, a solution obtained from our model may better hedge against uncertainty, thus leading to better ("expected") results than if a deterministic setting was assumed. 


\section{Conclusions}

In this work, we proposed a chance-constrained optimization model for the shelter site location problem as a means for capturing uncertainty in the demand. By invoking the central limit theorem we were able to obtain a non-linear deterministic equivalent model, which was reformulated in order to "isolate" the non-linear terms in a way that these can be approximated by a family of piecewise linear functions. Eventually, it was possible to obtain a mixed-integer linear programming model that can be tackled by an off-the-shelf solver when medium and large instances of the problem are considered. In particular, it was possible to solve the stochastic shelter site location problem at hand using data from the Kartal district in Istanbul, Turkey, as well as using a much larger set of data, namely the one associated with the whole Anatolian side of Istanbul.

The results show that different combinations of (i) shelter utilization rates, (ii) demand "service level", and (iii) demand pattern, may lead to completely different solutions. This is an indication that these aspects should be involved in a modeling framework for the problem. Moreover, comparisons were performed using a deterministic counterpart model. Again, clear differences were observed in the results. This supports the claim that capturing uncertainty in the shelter site location problem by means of probabilistic constraints may lead to solutions that can better hedge against uncertainty.

According to the approaches and results reported in this article, the contributions of this study can be summarized as follows: Demand uncertainty was included in the shelter site location problem, which resulted in a more realistic approach to be used in the preparedness phase of disaster management. The stochasticity is tackled by formulating a chance-constrained optimization model which requires an approximation approach using SOS2 variables. The relevance of the new modeling framework was evaluated by a series of computational tests.

This work represents a new research direction when it comes to handling the shelter site location problem. It also represents a contribution to the study of chance-constrained facility location problems under a maxmin objective.

One aspect that deserves future research has to do with the objective function. As we observed in the end of Section 5.2 the maxmin objective of the problem studied in this paper may easily render alternative optimal solutions as our intuition could foresee. However, when we look closely at these solutions, we immediately realize that they have different characteristics. This allows and even encourages a fine tuning in the decision making process via, for instance, a goal programming approach.

Another aspect that deserves future research is the possibility of embedding sheltering utilization balancing requirements in the probabilistic modeling framework proposed in this work. This poses new technical challenges when it comes to tackling the problem

Finally, another challenge emerging from the work done has to do with the assumption made about the demands. In this work, they are assumed to be independent. It would be interesting to investigate the case in which they are correlated.

\section{Acknowledgments}

This research has been partially supported by the Turkish Academy of Sciences and by the Portuguese Science Foundation, projects UID/MAT/04561/2013 (CMAF-CIO/FCUL) and UID/MAT/00297/2013 (CMA/FCT/UNL).

The authors wish to thank the anonymous referees for the valuable comments and suggestions provided.

\section{References}

Alçada-Almeida, L., Tralhão, L., Santos, L., \& Coutinho-Rodrigues, J. (2009). A multiobjective approach to locate emergency shelters and identify evacuation routes in urban areas. Geographical Analysis, 41, 9-29.

Altay, N., \& Green, W. G. (2006). OR/MS research in disaster operations management. European Journal of Operational Research, 175, 475-493.

Bayram, V., Tansel, B., \& Yaman, H. (2015). Compromising system and user interests in shelter location and evacuation planning. Transportation Research Part B: Methodological, 72, 146-163.

Bayram, V., \& Yaman, H. (2018a). Shelter location and evacuation route assignment under uncertainty: A benders decomposition approach. Transportation Science, 1-2. https://doi.org/10.1287/trsc.2017.0762, (Articles in Advance).

Bayram, V., \& Yaman, H. (2018b). A stochastic programming approach for shelter location and evacuation planning. RAIRO. doi:10.1051/ro/2017046.

Beale, E. M. L., \& Forrest, J. J. H. (1976). Global optimization using special ordered sets. Mathematical Programming, 10, 52-69.

Beale, E. M. L., \& Tomlin, J. A. (1970). Special facilities in a general mathematical programming system for non-convex problems using ordered sets of variables. In J. Lawrence (Ed.), Proceedings of the fifth international conference on operational research (pp. 447-454). London: Tavistock Publications.

Beraldi, P., Bruni, M. E., \& Conforti, D. (2004). Designing robust emergency medical service via stochastic programming. European Journal of Operational Research, 158, 183-193.

Bilsel, R. U., \& Ravindran, A. (2011). A multiobjective chance constrained programming model for supplier selection under uncertainty. Transportation Research Part B: Methodological, 45, 1284-1300.

Birge, J. R., \& Louveaux, F. V. (2011). Introduction to Stochastic Programming. Springer series in operations research and financial engineering (2nd ed.). New York: Springer-Verlag.

Caunhye, A. M., Nie, X., \& Pokharel, S. (2012). Optimization models in emergency logistics: A literature survey. Socio-Economic Planning Sciences, 46, 4-13.

Chanta, S., \& Sangsawang, O. (2012). Shelter-site selection during flood disaster. Lecture Notes in Management Science, 4, 282-288.

Charnes, A., \& Cooper, W. (1959). Chance constrained programming. Management Science, 6, 73-79.

Chen, A. Y., \& Yu, T.-Y. (2016). Network based temporary facility location for the emergency medical services considering the disaster induced demand and the transportation infrastructure in disaster response. Transportation Research Part B: Methodological, 91, 408-423.

Correia, I., \& Saldanha-da-Gama, F. (2015). Facility location under uncertainty. In G. Laporte, S. Nickel, \& F. Saldanha-da-Gama (Eds.), Location science (pp. 177-203). Berlin-Heidelberg: Springer.

Coutinho-Rodrigues, J., Tralhão, L., \& Alçada-Almeida, L. (2012). Solving a location-routing problem with a multiobjective approach: the design of urban evacuation plans. Journal of Transport Geography, 22, 206-218.

Elçi, O., Noyan, N., \& Bülbül, K. (2016). Chance-constrained stochastic programming under variable reliability levels with an application to humanitarian relief network design. http://research.sabanciuniv.edu/30556/1/SingleStageCC_ with_VariableRisk.pdf.

Espejo, I., Marìn, A., \& Rodrìguez-Chìa, A. (2012). Closest assignment constraints in discrete location problems. European Journal of Operational Research, 219, 49-58.

Galindo, G., \& Batta, R. (2013). Review of recent developments in OR/MS research in disaster operations management. European Journal of Operational Research, 230, 201-211.

Hong, X., Lejeune, M. A., \& Noyan, N. (2015). Stochastic network design for disaster preparedness. IIE Transactions, 47, 329-357.

Hoyos, M. C., Morales, R. S., \& Akhavan-Tabatabaei, R. (2015). OR models with stochastic components in disaster operations. Computers $\mathcal{E}$ Industrial Engineering, 82, 183-197.

Jeroslow, R. G., \& Lowe, J. K. (1984). Modelling with integer variables. Mathematical Programming Study, 22, 167-184.

Kılcı, F., Kara, B. Y., \& Bozkaya, B. (2015). Locating temporary shelter areas after an earthquake: A case for Turkey. European Journal of Operational Research, 243 323-332.

Kovács, G., \& Spens, K. M. (2007). Humanitarian logistics in disaster relief operations. International Journal of Physical Distribution \& Logistics Management, 37, 99-114.

Leiras, A., de Brito, I., Jr, Peres, E. Q., Bertazzo, T. R., \& Yoshizaki, H. T. Y. (2014). Literature review of humanitarian logistics research: Trends and challenges. Journal of Humanitarian Logistics and Supply Chain Management, 4, 95-130.

Li, A., Nozick, L., Xu, N., \& Davidson, R. (2012). Shelter location and transportation planning under hurricana conditions. Transportation Research Parte E: Logistics and Transportation Review, 48, 715-729.

Li, L., Jin, M., \& Zhang, L. (2011). Sheltering network planning and management with a case in the Gulf Coast region. International Journal of Production Economics, $131,431-440$.

Lin, C. (2009). Stochastic single-source capacitated facility location model with service level requirements. International Journal of Production Economics, 117, 439-451.

Murali, P., \& Ordónez, F. (2012). Facility location under demand uncertainty: response to a large-scale bio-terror attack. Socio-Economic Planning Sciences, 46, 78-87. 
Ortuño, M., Cristóbal, P., Ferrer, J., Martín-Campo, F., Muñoz, S., Tirado, G., \& Vitoriano, B. (2013). Decision aid models and systems for humanitarian logistics. A survey. In B. Vitoriano, J. Montero, \& D. Ruan (Eds.), Decision aid models for disaster management and emergencies (pp. 17-44). Paris: Atlantis Press.

Ozmen, B., Nurlu, M., Kuterdem, K., \& Temiz, A. (2010). Directorate of disaster operations. In Proceedings of the earthquake symposium (in Turkish). Kocaeli, Turkey.

Prékopa, A. (1995). Stochastic programming. Dordrecht, The Netherlands: Kluwer Academic Publishers.

Rawls, C. G., \& Turnquist, M. A. (2010). Pre-positioning of emergency supplies for disaster response. Transportation Research Part B: Methodological, 44, 521-534.

ReVelle, C. S., \& Hogan, K. (1989). The maximum availability location problem. Transportation Science, 23, 192-200.

Rojeski, P., \& ReVelle, C. S. (1970). Central facilities location under an investment constraint. Geographical Analysis, 2, 343-360.
Sherali, H. D., Carter, T. B., \& Hobeika, A. G. (1991). A location-allocation model and algorithm for evacuation planning under hurricane/flood conditions. Transportation Research Part B: Methodological, 25, 439-452.

Snyder, L. V. (2006). Facility location under uncertainty: A review. IIE Transactions, $38,537-554$.

Unal, G. (2010). A mathematical model based decision support system for emergency operations. Turkish Military Academy Defense Sciences Institutes Ph.D. Dissertation.

Wagner, J. L., \& Falkson, L. M. (1975). The optimal nodal location of public facilities with price-sensitive demand. Geographical Analysis, 7, 69-83.

Zhang, Z., \& Li, S. K. (2015). A novel probabilistic formulation for locating and sizing emergency medical service stations. Annals of Operations Research, 229, 813-835. 